\title{
A randomised clinical trial comparing myoinositol and metformin in PCOS
}

\author{
Kishan Chirania, Sujata Misra*, Sandhya Behera
}

Department of Obstetrics and Gynecology, SCB Medical College, Cuttack, Odisha, India

Received: 27 March 2017
Revised: 05 April 2017
Accepted: 08 April 2017

*Correspondence:

Dr. Sujata Misra,

E-mail: drsujatamisra@gmail.com

Copyright: (C) the author(s), publisher and licensee Medip Academy. This is an open-access article distributed under the terms of the Creative Commons Attribution Non-Commercial License, which permits unrestricted non-commercial use, distribution, and reproduction in any medium, provided the original work is properly cited.

\section{ABSTRACT}

Background: The medical management of PCOS is fast changing from the combination hormonal pills/progesterone for cycle regularisation, cosmetological treatment of acne and hirsutism to management of obesity and insulin resistance. This study evaluates the effects of the insulin sensitisers in improving the clinical and hormonal alterations in cases of PCOS and improving the reproductive outcomes.

Methods: This 3-arm prospective randomized comparative study was done from August 2015 to July 2016 in the Department of Obstetrics and Gynaecology at SCB MCH, Cuttack wherein patients of PCOS were studied based on treatment with metformin, myoinositol or both.

Results: Myoinositol helped in the resumption of spontaneous menstrual cycles in $66.66 \%$ of women with PCOS with menstrual complaints, whereas the same effect in patients who took metformin was only in $15.78 \%$, which was not significant. Use of myoinositol in Infertile women with PCOS resulted in a pregnancy in 57.14\% of women, without the need of any ovulation inducing agent while use of metformin gave a pregnancy in all the 9 patients, thought 5 out of them required clomiphene citrate for ovulation induction. With myoinositol there was a reduction in weight, BMI, LH/FSH ratio, acne and hirsutism. Metformin has resulted in a decrement of body weight, BMI and acne only.

Conclusions: This study conclusively proves that myoinositol has a definitive role in decreasing the ovarian dysfunction of PCOS. There has been a significant improvement in the symptom profile, weight loss and a significant change in the hormonal parameters.

Keywords: Insulin resistance, Metformin, Myoinositol

\section{INTRODUCTION}

PCOS is a common endocrine disturbance affecting woman of reproductive age. ${ }^{1}$ The recent concept supports lifestyle changes that promote weight loss which can essentially ameliorate the disease process in PCOS. ${ }^{2-4}$ Insulin sensitizers (metformin, glitazones) and anti-obesity drugs (sibutramine, orlistat etc) have been extensively studied. ${ }^{5}$

Myoinositol and berberine are the new-comers in the group of insulin sensitizers, supposedly safe due to their natural origin. ${ }^{6}$ While the diagnostic criteria for PCOS has stabilised, there has been a resurgence of newer drugs aimed at correcting the biochemical abnormalities and thereby, restoring ovarian function; compared to the traditional use of hormones for ovarian suppression and cycle regulation. $^{7}$

This study evaluates the effects of the insulin sensitisers in improving the clinical and hormonal alterations in cases of PCOS and improving the reproductive outcomes. ${ }^{8-10}$ It compares the effects of metformin and myoinositol alone, with another group of patients taking a combination of both the drugs. 


\section{METHODS}

This prospective study was done from August 2015 to July 2016 in the Department of Obstetrics and Gynaecology at SCB MCH, Cuttack.

Women presenting in the OBGYN OPD with irregular cycles or oligo/amenorrhea, infertility, hirsutism and excessive acne were evaluated. PCOS was diagnosed based on the rotterdam criteria. Both lean and obese PCOS patients were included in the study. Patients who were already diagnosed with PCOS and on treatment with the drugs compared in this study.

It is a 3-arm prospective randomized comparative study where patients were assigned into Group A, B and C based on computer generated numbers. It was an equivalence trial where the null hypothesis was that both myoinositol and metformin are equally effective in PCOS.

The sample size was calculated based on the formula,

$\mathrm{n}=\mathrm{Z}^{2} \times(\mathrm{p}) \times(1-\mathrm{p}) / \Delta^{2}$

$\mathrm{n}$ is the sample size.

$\mathrm{Z}$ is confidence interval i.e., 1.96 for $95 \%$

$\Delta$ is confidence level i.e., 0.05 for $\pm 5 \%$

$\mathrm{P}$ is the proportion of the population with the disease under study i.e, $5 \%$ which in decimal converts to 0.05 .

So, $\mathrm{n}=(1.96)^{2} \times(0.05) \times(1-0.05) /(0.05)^{2}$

$\mathrm{n}=72$

\section{Inclusion criteria}

The inclusion criteria for entry were

- Patient with oligomenorrhea/amenorrhea

- With polycystic ovaries on USG as described in rotterdam criteria

- With or without hyperandrogenism and/or obesity.

\section{Exclusion criteria}

- Abnormal TSH, PRL, AMH

- Any chronic illness in the past or present- TB, thyroid disease, malabsorption

- History of chemotherapy or radiotherapy in childhood.

After randomization, each group comprised of 24 patients each recieving the following treatment.

- Group A-Myoinositol 1g/day

- Group B-Metformin 1000mg/day
- Group C- Myoinositol 1g/day + Metformin 000mg/day

Since patients already on treatment with either myoinositol or metformin were also included in the study, the number of patients in each group slightly differed with group A having 26 patients, group B with 28 and group C with 22 patients. But the difference was not found to be statistically significant.

The treatment duration was 4 months. The patients were explained how to take the medicines, possible side effects and explained to come for follow-up.

Clinically, weight, height, waist/hip ratio and BP was measured along with examination for Hirsutism.

Ultrasound was done to diagnose PCOS, ovarian volume, endometrial thickness was noted. In the $3^{\text {rd }}$ and $4^{\text {th }}$ month, a mid-cycle ultrasound was done to look for spontaneous ovulation.

Investigations done at baseline were FSH, LH, TSH, PRL, fasting blood sugar, fasting insulin and serum lipid profile. The FSH, LH were done on the Day 2/Day 3 of a spontaneous or an induced menses.

At the end of $4^{\text {th }}$ month, FSH, LH, FBS, fasting insulin were repeated. Changes in weight and waist/hip ratio were noted clinically.

Ultrasound was done at the end of 4 months to look for any improvement in the ovaries in terms of the number of follicles and the ovarian volume. Transabdominal ultrasound was done in unmarried young patients and transvaginal ultrasound done for patients who presented with Infertility.

\section{RESULTS}

In the study $34.2 \%$ were in myoinositol group, $36.8 \%$ were in metformin group and $28.9 \%$ were in myoinositol+metformin group.

There was no significant difference in age between three groups ( $p$ value: 0.154 ) thus ensuring age matching.

On comparison of the symptoms at the initiation of treatment between the three groups, was no significant difference in symptom profile of subjects in all the three groups, ensuring adequate matching with respect to symptoms (p value: 0.244 )

Comparison of hirsutism before treatment between three groups was statistically significant with more number of subjects in the myoinositol+metformin group. ( $\mathrm{p}$ value: 0.038). Comparison of acne before treatment between three groups showed a $\mathrm{p}$ value of 0.412 ensuring that the patients were adequately matched in this respect. 
Table 1: Comparison of FSH, LH and LH/ FSH and comparison of Fasting insulin, FBS and FBS/insulin ratio between the three groups.

\begin{tabular}{|llllllll|l|} 
& \multicolumn{2}{l}{ Groups } & \multicolumn{2}{c}{ Metformin } & \multicolumn{3}{c|}{ Myoinositol+Metformin } \\
& Mean & SD & Mean & SD & \multicolumn{2}{c|}{ Mean } & SD & \\
\hline FSH & 5.63 & 0.97 & 5.14 & 1.55 & 5.83 & 1.31 & 0.160 \\
\hline LH & 13.43 & 6.68 & 12.59 & 9.47 & 14.87 & 3.49 & 0.539 \\
\hline LH/FSH & 2.33 & 0.98 & 2.44 & 1.68 & 2.64 & 0.77 & 0.682 \\
\hline F.Insulin & 13.55 & 5.03 & 18.70 & 7.42 & 17.30 & 6.72 & $0.015^{*}$ \\
\hline FBS & 90.69 & 9.51 & 86.51 & 25.03 & 93.82 & 12.77 & 0.342 \\
\hline FBS/Ins & 7.48 & 2.36 & 11.82 & 22.47 & 6.39 & 3.25 & 0.332 \\
\hline
\end{tabular}

p value for Fasting Insulin: 0.015

Table 2: Comparison of ovarian volume on the right and left side between the three groups.

\begin{tabular}{|llllllll|}
\hline & Groups & \multicolumn{3}{c}{} & \multicolumn{3}{c|}{ Meinositol + Metformin } \\
& Myoinositol & & \multicolumn{2}{c|}{ Metformin } & \multicolumn{3}{c|}{ Myoilue } \\
& Mean & SD & Mean & SD & Mean & SD & \\
\hline Ovarian Volume Right & 10.92 & 4.93 & 11.33 & 4.08 & 10.03 & 3.40 & 0.551 \\
\hline Ovarian Volume Left & 12.45 & 3.72 & 10.78 & 3.47 & 12.48 & 2.93 & 0.124 \\
\hline
\end{tabular}

$\mathrm{p}$ value: 0.551 and 0.124 for both ovaries

Table 3: Summary of patient profile and anthropometry.

\begin{tabular}{|c|c|c|c|c|}
\hline & Myoinositol, $n=26$ & Metformin, $\mathrm{n}=\mathbf{2 8}$ & Myoinositol+ Metformin, $\mathrm{n}=22$ & p value \\
\hline Age (years) & $23.92 \pm 3.70$ & $23.68 \pm 4.23$ & $21.9 \pm 3.45$ & 0.154 \\
\hline Amenorrhea (\%) & 46.2 & 53.6 & 54.5 & \multirow[b]{3}{*}{0.244} \\
\hline Infertility $(\%)$ & 53.8 & 32.1 & 36.4 & \\
\hline Irregular cycles $(\%)$ & 0 & 14.3 & 9.1 & \\
\hline Hirsutism (\%) & 46.2 & 57.1 & 81.8 & 0.038 \\
\hline Acne $(\%)$ & 23.1 & 39.3 & 36.4 & 0.412 \\
\hline Weight (kg) & $59.23 \pm 2.07$ & $61.79 \pm 5.92$ & $67.36 \pm 17.87$ & 0.05 \\
\hline BMI $\left(\mathrm{kg} / \mathrm{m}^{2}\right)$ & $24.63 \pm 3.32$ & $25.44 \pm 2.68$ & $25.02 \pm 9.14$ & 0.865 \\
\hline W/H Ratio & $0.81 \pm 0.04$ & $0.82 \pm 0.04$ & $0.03 \pm 0.559$ & 0.559 \\
\hline
\end{tabular}

Table 4: The laboratory features and ultrasound.

\begin{tabular}{|c|c|c|c|c|}
\hline Parameter & $\begin{array}{l}\text { Myoinositol } \\
(\mathrm{n}=26)\end{array}$ & $\begin{array}{l}\text { Metformin } \\
(n=28)\end{array}$ & $\begin{array}{l}\text { Myoinositol+Metformin } \\
(\mathrm{n}=22)\end{array}$ & p value \\
\hline FSH (U/L) & $5.63 \pm 0.97$ & $5.14 \pm 1.55$ & $5.83 \pm 1.31$ & 0.160 \\
\hline $\mathrm{LH}(\mathrm{U} / \mathrm{L})$ & $13.43 \pm 0.68$ & $12.59 \pm 9.47$ & $14.87 \pm 3.49$ & 0.539 \\
\hline LH/FSH Ratio & $2.33 \pm 0.98$ & $2.44 \pm 1.68$ & $2.64 \pm 0.77$ & 0.682 \\
\hline Fasting Insulin $\mu \mathrm{U} / \mathrm{ml}$ & $13.55 \pm 5.03$ & $18.7 \pm 7.42$ & $17.3 \pm 6.72$ & 0.015 \\
\hline FBS $(\mathrm{mg} \%)$ & $90.69 \pm 9.51$ & $86.51 \pm 25.03$ & $93.82 \pm 12.77$ & 0.342 \\
\hline FBS/Insulin & $7.48 \pm 2.36$ & $11.82 \pm 22.47$ & $6.39 \pm 3.25$ & 0.332 \\
\hline Ovarian volume-Right side (ml) & $10.92 \pm 4.93$ & $11.33 \pm 4.08$ & $10.03 \pm 3.40$ & 0.551 \\
\hline Ovarian volume-Left side (ml) & $12.45 \pm 3.72$ & $10.78 \pm 3.47$ & $12.48 \pm 2.93$ & 0.124 \\
\hline
\end{tabular}

Comparison of FSH, LH, LH/FSH showed a $\mathrm{p}$ value $>0.05$ in all the 3 parameters This ensures matching in selection of subjects with respect to hormonal levels. The mean fasting insulin was higher in metformin group (18.7 \pm 7.42$)$ than in other two groups.

$\mathrm{p}$ value for FBS and FBS/insulin ratio were 0.342 and 0.332 respectively between three groups. This ensures matching in selection of subjects with respect to the same (Table 1).

The comparison of ovarian volume on right and left side between three groups was not of any stastical significance (Table 2). On comparison of the patient profile and anthropometry in the three groups, only the $\mathrm{p}$ value for hirsutism: 0.038 (statistically significant). For all other 
parameters, there is no difference of statistical significance Table 3. On evaluation of the laboratory features and ultrasound amongst the three groups, only the $\mathrm{p}$ value for fasting insulin 0.015 was statistically significant Table 4.

The primary outcome of relief from menstrual complaints $\mathrm{p}$ value $<0.001$ and that for pregnancy rate $\mathrm{p}$ value $<0.001$ was statistically significant (Table 5).
In the metformin group, $\mathrm{p}$ value for relief from menstrual complaints: $>0.05$ and $p$ value for pregnancy rate $<0.001$ (Table 6). However, 5 patients out of 9 infertile patients in the metformin group had received clomiphene citrate for ovulation induction.

Table 5: Primary outcome measures in the myoinositol group $(n=26)$.

\begin{tabular}{|lllll|} 
& Before & After & Improvement (\%) & p value \\
Menstrual complaints & 12 & 4 & 66.66 & $<0.001$ \\
\hline Infertility & 14 & 6 & 57.14 & $<0.001$ \\
\hline
\end{tabular}

Table 6: Primary outcome measures in the metformin group $(n=28)$.

\begin{tabular}{|lllll|} 
& Before treatment & After treatment & Improvement $(\%)$ & p value \\
\hline Menstrual complaints & 19 & 16 & 15.78 & $>0.05$ \\
\hline Infertility & 9 & 0 & 100 & $<0.001$ \\
\hline
\end{tabular}

Table 7: Primary outcome measures with myoinositol+metformin group $(n=22)$.

\begin{tabular}{|lllll|} 
& Before treatment & After treatment & Improvement $(\%)$ & p value \\
\hline Menstrual complaints & 14 & 6 & 57.14 & $<0.001$ \\
\hline Infertility & 8 & 0 & 100 & $<0.001$ \\
\hline
\end{tabular}

Table 8: Secondary outcome measures in myoinositol group.

\begin{tabular}{|llllll|}
\hline & Before & After & & P value \\
\hline Weight & Mean & SD & Mean & SD & $<0.001^{*}$ \\
\hline BMI & 59.23 & 9.070 & 58.00 & 8.65 & $<0.001^{*}$ \\
\hline LH/FSH & 24.63 & 3.31 & 24.06 & 3.43 & $0.011^{*}$ \\
\hline F.Insulin & 2.32 & 0.98 & 2.17 & 0.92 & - \\
\hline Ovarian volume right & 13.55 & 5.02 & 13.55 & 5.02 & - \\
\hline Ovarian volume left & 10.92 & 4.93 & 10.92 & 4.93 & 0.095 \\
\hline
\end{tabular}

Table 9: Secondary outcome measures in metformin group.

\begin{tabular}{|llllll|}
\hline & Before & & After & & P value \\
\hline Weight & Mean & SD & Mean & SD & $0.003^{*}$ \\
\hline BMI & 61.79 & 5.92 & 61.04 & 6.11 & $0.004^{*}$ \\
\hline LH/FSH & 25.44 & 2.67 & 25.14 & 2.67 & - \\
\hline F.Insulin & 2.43 & 1.67 & 2.43 & 1.67 & - \\
\hline Ovarian volume right & 18.69 & 7.41 & 18.69 & 7.41 & - \\
\hline Ovarian volume left & 11.33 & 4.08 & 11.33 & 4.08 & - \\
\hline
\end{tabular}

Table 10: Secondary outcome measures in Myoinositol+Metformin group.

\begin{tabular}{|llllll|}
\hline & Before & & After & & P value \\
\hline Weight & Mean & SD & Mean & SD & $0.005^{*}$ \\
\hline BMI & 67.36 & 17.87 & 63.64 & 13.11 & 0.686 \\
\hline LH/FSH & 25.01 & 9.13 & 25.62 & 4.02 & 0.088 \\
\hline F.Insulin & 2.63 & 0.77 & 2.63 & 0.77 & $0.019^{*}$ \\
\hline Ovarian volume right & 17.29 & 6.71 & 15.78 & 5.69 & - \\
\hline Ovarian volume left & 10.02 & 3.39 & 10.02 & 3.39 & - \\
\hline
\end{tabular}


In the group on both drugs, the $\mathrm{p}$ value for relief of menstrual complaints was $<0.001$ and the $p$ value for pregnancy rate: $<0.001$ (statistically significant in both) Table 7.

Secondary outcome measures in myoinositol group showed significant $\mathrm{p}$ values for weight, BMI and LH/FSH. No significant effect was seen on fasting insulin and ovarian volume (Table 8).

Secondary outcome measures in metformin group $\mathrm{p}$ value for weight and BMI was statistically significant. No change was seen on LH/FSH, fasting insulin and ovarian volume on either sides (Table 9).

Secondary outcome measures in myoinositol+metformin group showed statistically significant difference in weight and fasting insulin. No significant effect was seen on BMI, LH/FSH ratio and ovarian volume on both sides (Table $10)$.

\section{DISCUSSION}

The primary outcome measures focussed in this study were i) resumption of spontaneous regular cycles in patients with oligo/amenorrhea and ii) pregnancy rate in infertile patients

The secondary outcome measures that were compared at enrolment and after treatment were:

- Clinical parameters-weight, BMI, acne and hirsutism

- Laboratory parameters-LH/FSH ratio, fasting insulin

- Ultrasound appearance of ovaries.

Patients in the 3 groups were adequately matched with respect to the age at presentation, symptoms, and complaints of acne. The patients were also matched in regard to anthropometric assessments like weight, height, BMI and $\mathrm{W} / \mathrm{H}$ ratio. There was also good matching in baseline FSH, LH, LH/FSH ratio, fasting blood sugar and ovarian volumes.

The only clinical parameter where there was a disparity was Hirsutism which was higher in the Group C. And the laboratory parameter where there was disparity in matching was in the fasting insulin levels between the 3 groups. The mean fasting insulin level was higher in the Group B compared to the other 2 groups. This did not make a difference in the final comparison since this was not one among the primary outcome.

In Group A $(\mathrm{n}=26)$ who were treated with myoinositol $1 \mathrm{~g} /$ day, $66.66 \%$ ( $\mathrm{p}<0.001$ ) patients got relieved from menstrual complaints and $57.14 \%(\mathrm{p}<0.001)$ of patients with Infertility reported a pregnancy.

Further, in Group A, there was a statistically significant reduction in the body weight and BMI, LH/FSH Ratio, hirsutism and acne in the treated patients. No change was demonstrated in fasting insulin levels and ovarian volume.

In Group B $(\mathrm{n}=28)$ in which patients received $1 \mathrm{~g}$ metformin per day, only $15.78 \%$ ( $p>0.05)$ patients with menstrual complaints had improvement, whereas all patients with Infertility reported a pregnancy $(p<0.001)$. But 5 out of the 9 patients of infertility had taken clomiphene citrate for ovulation induction. This factor is a confounding factor in the interpretation of this result.

A significant decrement was demonstrated only in body weight, BMI and acne. There was no change in hirsutism, $\mathrm{LH} / \mathrm{FSH}$ ratio, fasting insulin and ovarian volume.

In Group $\mathrm{C}(\mathrm{n}=22)$, patients received both myoinositol and metformin $1 \mathrm{~g}$ each per day. 57.14\% (p<0.001) patients with menstrual complaints resumed spontaneous regular cycles and all patients with infertility reported a pregnancy $(\mathrm{p}<0.001)$.

There was a statistically significant reduction in body weight, fasting insulin and acne. There was no change in hirsutism and ovarian volume. None of the patients in any of the 3 groups reported any major side effects.

None of the patients required discontinuation of the drug due to any intolerable side effects. The expense of treatment was a concern for some patients in Group A (myoinositol) and Group C (myoinositol+metformin). Complaints of 4 patients in the metformin group was nausea which got relieved after advising to take the drug with meals. 5 patients in myoinositol reported an unpleasant taste of the powder form of the drug when dissolved in water and taken. They were advised the tablet form.

Use of both of the insulin sensitizers myoinositol and metformin has resulted in significant changes in the symptoms of PCOS in this study, which reflects that a change in the pathogenesis has been caused by these drugs. The use of either drug or in combination in this study has shown a significant weight loss. And since the time that lifestyle factors have been implicated in PCOS, weight loss has been greatly emphasised in its management. ${ }^{11,12}$

The regular use of myoinositol helped in the resumption of spontaneous menstrual cycles in $66.66 \%$ of women with PCOS with menstrual complaints, whereas the same effect in patients who took metformin was only in $15.78 \%$, which was not significant. Use of myoinositol in Infertile women with PCOS resulted in a pregnancy in $57.14 \%$ of women, without the need of any ovulation inducing agent. ${ }^{13}$ The role of myoinositol in improving the oocyte quality cannot be overlooked, the fact that has been proven in studies. ${ }^{14,15}$

Also, the not so rare risks of ovarian hyperstimulation syndrome (OHSS) and multiple pregnancy are also absent when an ovulation inducing agent is not used in PCOS. ${ }^{16,17}$ 
Metformin use in women with PCOS with Infertility in my study gave a pregnancy in all the 9 patients, but 5 out of them required clomiphene citrate for ovulation induction. Hence, the actual pregnancy rate in the group for metformin alone could not be assessed since clomiphene was the confounding factor.

With regard to the secondary outcome measures, myoinositol has resulted in a significant reduction of more parameters than metformin. With myoinositol there was a reduction in weight, $\mathrm{BMI}$, $\mathrm{LH} / \mathrm{FSH}$ ratio, acne and hirsutism. Metformin has resulted in a decrement of body weight, BMI and acne only. This finding perhaps supports the hypothesis that myoinositol works at the ovarian level and brings about a change in the hormonal mileu, decreases the LH/FSH ratio and hence improves the oocyte quality. ${ }^{17}$

The above-mentioned findings show a more favourable response of PCOS women to myoinositol compared to metformin. But more studies with a bigger sample size and longer follow-up are required to establish the accurate results.

When used in Infertility, an optimum ratio of myoinositol and D-chiro-inositol of 40:1 has been recommended by recent studies which is said to improve oocyte quality. ${ }^{18}$

\section{CONCLUSION}

This study conclusively proves that myoinositol has a definitive role in decreasing the ovarian dysfunction of PCOS. There has been a significant improvement in the symptom profile, weight loss and a significant change in the hormonal parameters.

In the combination group, the results obtained are similar to that of myoinositol alone. And when compared to the results obtained with metformin alone, it seems likely that the results obtained in the combination group could be due to the myoinositol component. Results have been fairs with a dose as low as $1 \mathrm{~g}$ per day. No adverse effects have been reported by patients in this study.

Myoinositol can be tried as an ovulation induction agent in infertile PCOS patients. Myoinositol promotes monofollicular development. This precludes the risk of OHSS and multiple pregnancies that is seen with clomiphene, gonadotropins.

Funding: No funding sources

Conflict of interest: None declared

Ethical approval: The study was approved by the Institutional Ethics Committee

\section{REFERENCES}

1. Sirman SM, Pate KA. Epidemiology, diagnosis, and management of polycystic ovary syndrome. Clin Epidemiol. 2013(18);6:1-13.
2. Liang SJ, Liou TH, Lin HW, Hsu CS, Tzeng CR, Hsu MI. Obesity is the predictor of impaired glucose tolerance and metabolic disturbance in polycystic ovary syndrome. Acta Obstet Gynecol Scand. 2012;91(10):1167-72.

3. Fritz MA, Speroff L. Clinical Gynecologic Endocrinology and Infertility, 8th edition (Lippincott Williams and Wilkins; 2010:505.

4. Ravn P, Haugen AG, Glintborg D. Overweight in polycystic ovary syndrome. An update on evidence based advice on diet, exercise and metformin use for weight loss. Minerva Endocrinol. 2013;38(1):59-76.

5. Palomba S, Falbo A, La Sala GB. Metformin and gonadotropins for ovulation induction in patients with polycystic ovary syndrome: a systematic review with meta-analysis of randomized controlled trials. Reprod Biol Endocrinol. 2014(3);12:3.

6. Nordio M, Proietti E. The combined therapy with myo-inositol and D-chiro-inositol reduces the risk of metabolic disease in PCOS overweight patients compared to myo-inositol supplementation alone. Eur Rev Med Pharmacol Sci. 2012;16(5):575-81.

7. Lujan ME, Chizen DR, Pierson RA. Diagnostic criteria for polycystic ovary syndrome: pitfalls and controversies. J Obstet Gynaecol Can. 2008;30(8):671-9.

8. Carmina E, Lobo RA. Do hyperandrogenic women with normal menses have polycystic ovary syndrome? Fertil Steril. 1999;71:319-22.

9. O'Reilly MW, Taylor AE, Crabtree NJ, Hughes BA, Capper F, Crowley RK et al. Hyperandrogenemia predicts metabolic phenotype in polycystic ovary syndrome: the utility of serum androstenedione. J Clin Endocrinol Metab. 2014;99(3):1027-36.

10. Vittorio U, Giuseppina P. Updates on the myo-inositol plus D-chiro-inositol combined therapy in polycystic ovary syndrome. Expert Rev Clin Pharmacol. 2014 Sep;7(5):623-31.

11. Ciotta L, Stracquadanio M, Pagano I, Carbonaro A, Palumbo M, Gulino F. Effects of myo-inositol supplementation on oocyte's quality in PCOS patients: a double blind trial. Eur Rev Med Pharmacol Sci. 2011;15(5):509-14.

12. Carlomagano G, Unfer V. Inositol safety: clinical evidences. Eur Rev Med Pharmacol Sci. 2011;15:9316.

13. Le Donne M, Alibrandi A, Giarrusso R, Lo Monaco I, Muraca U. Diet, metformin and inositol in overweight and obese women with polycystic ovary syndrome: effects on body composition. Minerva Ginecol. 2012;64(1):23-9.

14. Costantino D, Minozzi G, Minozzi E, Guaraldi C. Metabolic and hormonal effects of Myoinositol in women with Polycystic Ovary Syndrome: a doubleblind trial. Eur Rev Med Pharmacol Sci. 2009;13(2):105-10.

15. Papaleo E, Unfer V, Baillargeon JP, De Santis L, Fusi $\mathrm{F}$, Brigante $\mathrm{C}$ et al. Myo-inositol in patients with polycystic ovary syndrome: A novel method for 
ovulation induction. Gynecol Endocrinol. 2007;23(12):700-3.

16. Gerli S, Papaleo E, Ferrari A, Di Renzo GC. Effects of Inositol on ovarian function and metabolic factors in women with PCOS: a randomized double blind placebo-controlled trial. Eur Rev Med Pharmacol Sci. 2007;11(5):347-54.

17. Raffone E, Rizzo P, Benedetto V. Insulin sensitiser agents alone and in co-treatment with r-FSH for ovulation induction in PCOS women. Gynecol Endocrinol. 2010;26(4):275-80.

18. Carlomagno G, Unfer V, Roseff S. The D-chiroinositol paradox in the ovary. Fertil Steril. 2011;95(8):2515-6.

Cite this article as: Chirania $\mathrm{K}$, Misra S, Behera S. A randomised clinical trial comparing myoinositol and metformin in PCOS. Int J Reprod Contracept Obstet Gynecol 2017;6:1814-1820. 